\title{
NON-SUPERREFLEXIVITY OF GARLING SEQUENCE SPACES AND APPLICATIONS TO THE EXISTENCE OF SPECIAL TYPES OF CONDITIONAL BASES
}

\author{
FERNANDO ALBIAC, JOSÉ LUIS ANSORENA, STEPHEN J. DILWORTH, \\ AND DENKA KUTZAROVA
}

\begin{abstract}
In this paper we settle in the negative the problem of the superreflexivity of Garling sequence spaces by showing that they contain a complemented subspace isomorphic to a non superreflexive mixed-norm sequence space. As a by-product of our work, we give applications of this result to the study of conditional Schauder bases and conditional almost greedy bases in this new class of Banach spaces.
\end{abstract}

\section{InTRODUCTION AND BACKGROUND}

Suppose $1 \leq p<\infty$ and let $\mathbf{w}=\left(w_{j}\right)_{j=1}^{\infty}$ belong to the set of weights

$$
\mathcal{W}:=\left\{\left(w_{j}\right)_{j=1}^{\infty} \in c_{0} \backslash \ell_{1}: 1=w_{1} \geq w_{2}>\cdots w_{j} \geq w_{j+1} \geq \cdots>0\right\} .
$$

The Garling sequence space, denoted $g(\mathbf{w}, p)$, is the Banach space consisting of all scalar sequences $f=\left(a_{j}\right)_{j=1}^{\infty}$ such that

$$
\|f\|_{g(\mathbf{w}, p)}=\sup _{\phi \in \mathcal{O}}\left(\sum_{n=1}^{\infty}\left|a_{\phi(j)}\right|^{p} w_{j}\right)^{1 / p}<\infty,
$$

where $\mathcal{O}$ denotes the set of all increasing functions from $\mathbb{N}$ to $\mathbb{N}$.

The study of the isomorphic structure of these spaces, which generalize an example of Garling from [16], has been recently initiated in [6]. For expositional ease, we have gathered in Theorem 1.1 a few geometric properties of $g(\mathbf{w}, p)$ that will help the reader to contextualize the results herein.

2010 Mathematics Subject Classification. 46B45, 46B25, 46B15, 46B10, 46B07, $41 \mathrm{~A} 65$.

Key words and phrases. subsymmetric basis, Garling spaces, sequence spaces, superreflexivity, Besov spaces, conditional bases, conditionality constants, almost greedy bases, complemented subspaces.

F. Albiac and J. L. Ansorena acknowledge the support of the grant MTM201453009-P (MINECO, Spain). F. Albiac was also supported by the grant MTM201676808-P (MINECO, Spain). Add Steve's and Denka's Grant Support. 
Theorem 1.1 (see $[5,6]$ ). Let $1 \leq p<\infty$ and $\mathbf{w} \in \mathcal{W}$. Then:

(a) The unit vector system $\left(\mathbf{e}_{j}\right)_{j=1}^{\infty}$ is a 1-subsymmetric basis of $g(\mathbf{w}, p)$ which is not symmetric.

(b) Any subsymmetric basis of $g(\mathbf{w}, p)$ is equivalent to $\left(\mathbf{e}_{j}\right)_{j=1}^{\infty}$.

(c) $g(\mathbf{w}, p)$ is reflexive if and only if $p>1$.

(d) For every $\varepsilon>0$ and every infinite dimensional subspace $\mathbb{Y}$ of $g(\mathbf{w}, p)$ there is a further subspace $\mathbb{Z} \subseteq \mathbb{Y}$ that is $(1+\varepsilon)$ isomorphic to $\ell_{p}$ and $(1+\varepsilon)$-complemented in $g(\mathbf{w}, p)$.

Garling sequence spaces can be regarded as the subsymmetric counterpart of Lorentz sequence spaces $d(\mathbf{w}, p)$, consisting of all scalar sequences $f=\left(a_{n}\right)_{n=1}^{\infty}$ such that

$$
\|f\|_{d(\mathbf{w}, p)}=\sup _{\sigma \in \Pi}\left(\sum_{n=1}^{\infty}\left|a_{\sigma(n)}\right|^{p} w_{n}\right)^{1 / p}<\infty,
$$

where $\Pi$ is the set of permutations of $\mathbb{N}$. The study of the geometrical properties of the spaces $d(\mathbf{w}, p)$ were initiated in the early 1970's by Altshuler et al. [12]. Comparing Theorem 1.1 with the corresponding results in [12] reflects the fact that Garling sequence spaces behave to some extent similarly to Lorentz sequence spaces replacing symmetry with subsymmetry. Subsequently to [12], Altshuler [10] showed that $d(\mathbf{w}, p)$ is superreflexive if and only if $p>1$ and the weight $\mathbf{w}=\left(w_{j}\right)_{j=1}^{\infty}$ is regular, i.e,

$$
\sup _{m} \frac{1}{m w_{m}} \sum_{j=1}^{m} w_{j}<\infty,
$$

As for Garling spaces, a first attempt to determine whether or not the spaces $g(\mathbf{w}, p)$ were superreflexive was undertaken in [7]. Using nonlinear tools from approximation theory such as the fundamental function of the canonical basis the authors showed that $g(\mathbf{w}, p)$ fails to be superreflexive if the weight $\mathbf{w}$ is not regular. In this note we adopt a radically different, intrinsic approach, in the sense that the methods we use fall within the linear category. To be precise, in Section 2 we study in detail the complemented subspaces of $g(\mathbf{w}, p)$, and having done the groundwork we settle in the negative the problem of the superreflexivity of Garling sequence spaces in all cases by proving the following theorem, which exhibits an important structural difference between Lorentz spaces and Garling spaces.

Theorem 1.2. The Banach space $g(\mathbf{w}, p)$ is not superreflexive for any $1 \leq p<\infty$ and any $\mathbf{w} \in \mathcal{W}$. 
Garling sequence spaces can be regarded as the subsymmetric counterpart of Lorentz sequence spaces $d(\mathbf{w}, p)$, consisting of all scalar sequences $f=\left(a_{n}\right)_{n=1}^{\infty}$ such that

$$
\|f\|_{d(\mathbf{w}, p)}=\sup _{\sigma \in \Pi}\left(\sum_{n=1}^{\infty}\left|a_{\sigma(n)}\right|^{p} w_{n}\right)^{1 / p}<\infty,
$$

where $\Pi$ is the set of permutations of $\mathbb{N}$. The spaces $d(\mathbf{w}, p)$ were thoroughly investigated by Altshuler, Casazza and Lin in the early 1970's in the papers [12,13]. Subsequently, Altshuler showed in [10] that $d(\mathbf{w}, p)$ is superreflexive if and only if $p>1$ and the weight $\mathbf{w}=$ $\left(w_{j}\right)_{j=1}^{\infty}$ is regular. Recall that a weight is said to be regular if

$$
\sup _{m} \frac{1}{m w_{m}} \sum_{j=1}^{m} w_{j}<\infty
$$

A first attempt to determine whether or not the spaces $g(\mathbf{w}, p)$ were superreflexive was undertaken in [7]. A Banach space $\mathbb{X}$ is called superreflexive if every Banach space finitely representable in $\mathbb{X}$ is reflexive. Using nonlinear tools from approximation theory such as the fundamental function of the canonical basis, the authors showed that $g(\mathbf{w}, p)$ fails to be superreflexive if the weight $\mathbf{w}$ is not regular. Recall that a weight $\mathbf{w}=\left(w_{j}\right)_{j=1}^{\infty}$ is said to be regular if

$$
\sup _{m} \frac{1}{m w_{m}} \sum_{j=1}^{m} w_{j}<\infty,
$$

and is said to be bi-regular if both $\mathbf{w}$ and its conjugate weight $\mathbf{w}^{*}=$ $\left(1 /\left(j w_{j}\right)\right)_{j=1}^{\infty}$ are regular.

In this note we adopt a radically different, intrinsic approach, in the sense that the methods we use fall within the linear category. To be precise, in Section 2 we study in detail the complemented subspaces of $g(\mathbf{w}, p)$, and having done the groundwork we settle in the negative the problem of the superreflexivity of Garling sequence spaces in all cases by proving the following theorem:

Theorem 1.3. The Banach space $g(\mathbf{w}, p)$ is not superreflexive for any $1 \leq p<\infty$ and any $\mathbf{w} \in \mathcal{W}$.

Hence, while comparing Theorem 1.1 with the corresponding results in [12] reflects the fact that Garling sequence spaces behave to some extent similarly to Lorentz sequence spaces replacing symmetry with subsymmetry, Theorem 1.3 exhibits an important structural difference between these two types of spaces. 
In Section 3 we will apply the results from Section 2 to investigate the existence of conditional bases with some special features in $g(\mathbf{w}, p)$, which bridges our results with the theory of greedy approximation in Banach spaces.

Standard Banach space notation and terminology are used throughout (see [9]). For clarity, however, we record the notation that is used most heavily. We write $\mathbb{F}$ for the real or complex scalar field. Given a set of indices $I$, we denote by $\left(\mathbf{e}_{i}\right)_{i \in I}$ the unit vector system of $\mathbb{F}^{I}$, i.e., $\mathbf{e}_{i}=\left(\delta_{i, j}\right)_{j \in I}^{\infty}$, were $\delta_{i, j}=1$ if $i=j$ and $\delta_{i, j}=0$ otherwise. If $a_{j}$ are elements in a vector space, we will use the convention $\sum_{j=1}^{0} a_{j}=0$.

Given families of non-negative real numbers $\left(\alpha_{i}\right)_{i \in I}$ and $\left(\beta_{i}\right)_{i \in I}$ and $0<C<\infty$ the symbol $\alpha_{i} \lesssim_{C} \beta_{i}$ for $i \in I$ means that $\alpha_{i} \leq C \beta_{i}$ for all $i \in I$, while $\alpha_{i} \approx_{C} \beta_{i}$ for $i \in I$ means that $\alpha_{i} \lesssim_{C} \beta_{i}$ and $\beta_{i} \lesssim_{C} \alpha_{i}$ for $i \in I$. Now suppose $\left(\mathbf{x}_{n}\right)_{n=1}^{\infty}$ and $\left(\mathbf{y}_{n}\right)_{n=1}^{\infty}$ are basic sequences in $\mathbb{X}$ and $\mathbb{Y}$, respectively. We say that $\left(\mathbf{y}_{n}\right)_{n=1}^{\infty} C$-dominates $\left(\mathbf{x}_{n}\right)_{n=1}^{\infty}$ and write $\left(\mathbf{x}_{n}\right)_{n=1}^{\infty} \lesssim_{C}\left(\mathbf{y}_{n}\right)_{n=1}^{\infty}$ if $\left\|\sum_{n=1}^{\infty} a_{n} \mathbf{x}_{n}\right\|_{X} \lesssim_{C}\left\|\sum_{n=1}^{\infty} a_{n} \mathbf{y}_{n}\right\|_{Y}$ for all $\left(a_{n}\right)_{n=1}^{\infty} \in c_{00}$. Whenever $\left(\mathbf{x}_{n}\right)_{n=1}^{\infty} \lesssim_{C}\left(\mathbf{y}_{n}\right)_{n=1}^{\infty}$ and $\left(\mathbf{y}_{n}\right)_{n=1}^{\infty} \lesssim_{C}\left(\mathbf{x}_{n}\right)_{n=1}^{\infty}$, we say that $\left(\mathbf{x}_{n}\right)_{n=1}^{\infty}$ and $\left(\mathbf{y}_{n}\right)_{n=1}^{\infty}$ are $C$-equivalent, and write $\left(\mathbf{x}_{n}\right)_{n=1}^{\infty} \approx_{C}$ $\left(\mathbf{y}_{n}\right)_{n=1}^{\infty}$. In all the above cases, when the value of the constant $C$ is irrelevant, we simply drop it from the notation.

The norm of a linear operator from $T$ from a Banach space $\mathbb{X}$ into a Banach space $\mathbb{Y}$ by $\|T: \mathbb{X} \rightarrow \mathbb{Y}\|$. Given a basis $\mathcal{B}=\left(\mathbf{x}_{j}\right)_{j=1}^{\infty}$ for a Banach space $\mathbb{X}$ the support of $f=\sum_{j=1}^{\infty} a_{j} \mathbf{x}_{j} \in \mathbb{X}$ with respect to $\mathcal{B}$ is the set $\operatorname{supp}(f)=\left\{j: a_{j} \neq 0\right\}$. The coordinate projection on a set $A$ will be denoted by $S_{A}[\mathcal{B}, \mathbb{X}]$ or, if $\mathcal{B}$ and $\mathbb{X}$ are clear from context, $S_{A}$. Given $1 \leq p<\infty,\left(\oplus_{n=1}^{\infty} \mathbb{X}_{n}\right)_{n=1}^{\infty}$ denotes the direct sum in the $\ell_{p}$ sense of the sequence of Banach spaces $\left(\mathbb{X}_{n}\right)_{n=1}^{\infty}$.

More specialized notions from Banach space theory or approximation theory will be introduced as needed.

\section{Complemented subspaces of Garling Sequence spaces}

Theorem 1.3 will be a consequence of the following result, which trivially implies that $\ell_{\infty}$ is finitely representable in any Garling sequence space.

Theorem 2.1. Let $1 \leq p<\infty$ and $\mathbf{w} \in \mathcal{W}$. For each $\varepsilon>0$ there is a sublattice $Z \subseteq g(\mathbf{w}, p)$ that is $(1+\varepsilon)$-lattice complemented in $g(\mathbf{w}, p)$ and $(1+\varepsilon)$-lattice isomorphic to $\left(\oplus_{n=1}^{\infty} \ell_{\infty}^{n}\right)_{p}$.

In turn, the proof of Theorem 2.1 relies on Lemmas 2.2 and 2.5 below. The former is elementary and exhibits a "gliding hump" behaviour of the weights in $\mathcal{W}$. 
Lemma 2.2. Let $\left(w_{j}\right)_{j=1}^{\infty}$ be a non-increasing sequence of positive numbers with $\sum_{j=1}^{\infty} w_{j}=\infty$. For every $m \geq 0$ we have

$$
\lim _{k} \frac{\sum_{j=m+1}^{m+k} w_{j}}{\sum_{j=1}^{k} w_{j}}=1 \text {. }
$$

Proof. Since w is non-increasing,

$$
A:=\underset{k}{\limsup } \frac{\sum_{j=m+1}^{m+k} w_{j}}{\sum_{j=1}^{k} w_{j}} \leq 1,
$$

and, since $\lim _{k} \sum_{j=1}^{k} w_{j}=\infty$,

$$
B:=\liminf _{k} \frac{\sum_{j=m+1}^{m+k} w_{j}}{\sum_{j=1}^{k} w_{j}}=\liminf _{k} \frac{\sum_{j=1}^{m+k} w_{j}}{\sum_{j=1}^{k} w_{j}} \geq 1 .
$$

Since $B \leq A$, we obtain $A=B=1$.

In order to state and prove the following lemmata, it is convenient to set some notation. Given a tuple $f=\left(a_{j}\right)_{j=1}^{k}$ and $m \in \mathbb{N} \cup\{0\}$, let us define the sequence $\mathrm{I}_{m}(f)$ in $c_{00}$ by

$$
\mathrm{I}_{m}(f)=(\underbrace{0, \ldots, 0}_{m}, a_{1}, \ldots, a_{j}, \ldots, a_{k}, 0, \ldots, 0, \ldots) .
$$

Note that $\mathrm{I}=\mathrm{I}_{0}$ is the natural embedding of $\cup_{k=1}^{\infty} \mathbb{F}^{k}$ into $\mathbb{F}^{\mathbb{N}}$. We define

$$
\|f\|_{g}:=\|\mathrm{I}(f)\|_{g}, \quad f \in \cup_{k=1}^{\infty} \mathbb{F}^{k} .
$$

By the 1-subsymmetry of the unit vector basis we have $\left\|\mathrm{I}_{m}(f)\right\|_{g}=\|f\|_{g}$ for every tuple $f$ and every $m \in \mathbb{N}$.

Lemma 2.3. Let $1 \leq p<\infty$ and $\mathbf{w} \in \mathcal{W}$. Given $0<\varepsilon<1$ and tuples $f$ and $g$ with $\|f\| \leq 1$, there is a tuple $h$ such that $\|(h, f)\|_{g} \leq 1$ and $\|(g, h)\| \geq\left(\|g\|^{p}+1-\varepsilon\right)^{1 / p}$. Moreover, $h$ can be chosen to be a constant-coefficient $k$-tuple with $k$ as large as wished.

Proof. Let $f=\left(a_{j}\right)_{j=1}^{n}$ and $g=\left(b_{j}\right)_{j=1}^{m}$. Put $a_{j}=0$ for $j>n$ and define for every non-negative integer $k$,

$$
v_{k}=\sup _{\phi \in \mathcal{O}} \sum_{j=1}^{\infty}\left|a_{\phi(j)}\right|^{p} w_{j+k} .
$$

Since $\mathbf{w}$ is non-increasing we have $v_{k} \leq\|f\|_{g}^{p} \leq 1$ and

$$
v_{k} \leq\left(\sum_{i=1}^{n}\left|a_{i}\right|\right) w_{1+k}
$$

Hence, $\lim _{k} v_{k}=0$ since $\mathbf{w} \in c_{0}$. For any $n \in \mathbb{N}$ put 
6 F. AlBiAC, J. L. ANSORENA, S. J. DILWORTH, AND DENKA KUTZAROVA

For any nonnegative integer $k$ put

$$
\alpha_{k}=\frac{1-v_{k}}{\sum_{j=1}^{k} w_{j}} \in[0, \infty) .
$$

By Lemma 2.2, $\lim _{k} \alpha_{k} \sum_{j=m+1}^{m+k} w_{j}=1$, and since $\mathbf{w} \notin \ell_{1}, \lim _{k} \alpha_{k}=0$. We infer that there is $k \in \mathbb{N}$, which can be chosen larger than a given integer, such that

- $\alpha_{k} \sum_{j=m+1}^{m+k} w_{j} \geq 1-\varepsilon$ and

- $\alpha_{i} \geq \alpha_{k}$ whenever $1 \leq i \leq k$.

Let $h$ be the constant $k$-tuple whose entries are equal to $\alpha_{k}^{1 / p}$. We have

$$
\|(h, f)\|_{g}^{p}=\max _{0 \leq i \leq k} v_{i}+\alpha_{k} \sum_{j=1}^{i} w_{j} \leq \max _{i \geq 0} v_{i}+\alpha_{i} \sum_{j=1}^{i} w_{j}=1,
$$

and

$$
\|(g, h)\|_{g}^{p}=\|g\|^{p}+\alpha_{k} \sum_{j=m+1}^{m+k} w_{j} \geq\|g\|^{p}+1-\varepsilon .
$$

We will obtain Lemma 2.5 by using the full power of Lemma 2.3. Given $k \in \mathbb{N}$ we will denote by $\mathbf{v}[k]$ the positive constant-coefficient $k$-tuple whose norm in $g(\mathbf{w}, p)$ is one, i.e.,

$$
\mathbf{v}[k]=\frac{1}{\left(\sum_{j=1}^{k} w_{j}\right)^{1 / p}}(\underbrace{1, \ldots, 1}_{k}) .
$$

Lemma 2.4. Let $1 \leq p<\infty$ and $\mathbf{w} \in \mathcal{W}$. Given $k_{0} \in \mathbb{N}, t>1$ and $a$ tuple $f$ with $\|f\|_{g} \leq t$, there is $k \geq k_{0}$ such that $\|(\mathbf{v}[k], f)\|_{g} \leq t$.

Proof. Let $\varepsilon=1-t^{-p}$. Applying Lemma 2.3 with $g=0$ yields $k \geq k_{0}$ and a constant $k$-tuple $h$ verifying

$$
\left\|\left(h, t^{-1} f\right)\right\|_{g} \leq 1 \text { and } s:=\|h\|_{g} \geq(1-\varepsilon)^{1 / p}=t^{-1} .
$$

Notice that $h=s \mathbf{v}[k]$. Using the 1-unconditionality of the unit vector basis of $g(\mathbf{w}, p)$ we obtain

$$
\|(\mathbf{v}[k], f)\|_{g}=t\left\|\left((s t)^{-1} h, t^{-1} f\right)\right\|_{g} \leq t\left\|\left(h, t^{-1} f\right)\right\|_{g} \leq t,
$$

as desired.

Given a tuple $\kappa=\left(k_{i}\right)_{i=1}^{n}$ we put

$$
\mathbf{v}[\kappa]=\mathbf{v}\left[k_{1}, \ldots, k_{i}, \ldots k_{n}\right]=\left(\mathbf{v}\left[k_{1}\right], \ldots, \mathbf{v}\left[k_{i}\right], \ldots, \mathbf{v}\left[k_{n}\right]\right) .
$$


Lemma 2.5. Let $1 \leq p<\infty$ and $\mathbf{w} \in \mathcal{W}$. Given $0<\varepsilon<1$ and $k, n \in \mathbb{N}$ there is a sequence $\kappa=\left(k_{i}\right)_{i=1}^{n}$ such that $k_{i} \geq k$ for $i=1, \ldots$, $n$ and $\|\mathbf{v}[\kappa]\|_{g} \leq 1+\varepsilon$.

Proof. Lemma 2.4 allows us to recursively construct a sequence $\left(q_{i}\right)_{i=1}^{\infty}$ in $\mathbb{N}$ such that $q_{1}=k, q_{i} \geq k$ for all $i \in \mathbb{N}$, and

$$
\left\|\mathbf{v}\left[q_{n}, \ldots, q_{i}, \ldots, q_{1}\right]\right\|_{g} \leq(1+\varepsilon)^{(n-1) / n}
$$

for all $n \in \mathbb{N}$. To finish the proof we just need to take $\kappa=\left(q_{n+1-i}\right)_{i=1}^{n}$ for a given $n \in \mathbb{N}$.

Here and from now on we use the convention $\sum_{i=1}^{0} a_{j}=0$. Given an increasing sequence $\gamma=\left(k_{n}\right)_{n=1}^{\infty}$ of natural numbers, define $q(\gamma)=$ $\left(q_{n}\right)_{n=0}^{\infty}$ by $q_{n}=\sum_{i=1}^{n} k_{n}$ and $P_{\gamma}: \mathbb{F}^{\mathbb{N}} \rightarrow \mathbb{F}^{\mathbb{N}}$ by

$$
f=\left(a_{j}\right)_{j=1}^{\infty} \mapsto P_{\gamma}(f)=\left(\frac{\left(\sum_{j=1}^{q_{n}-q_{n-1}} w_{j}\right)^{1 / p}}{\sum_{j=1+q_{n-1}}^{q_{n}} w_{j}} \sum_{j=1+q_{n-1}}^{q_{n}} a_{j} w_{j}\right)_{n=1}^{\infty} .
$$

Lemma 2.6. let $\gamma$ be a sequence o natural numbers and $t \in(0, \infty)$. Assume that, if $q(\gamma)=\left(q_{n}\right)_{n=0}^{\infty}$,

$$
\frac{\sum_{j=1}^{q_{n}-q_{n-1}} w_{j}}{\sum_{j=1+q_{n-1}}^{q_{n}} w_{j}} \geq t
$$

for all $n \in \mathbb{N}$. Then $\left\|P_{\gamma}: g(\mathbf{w}, p) \rightarrow \ell_{p}\right\| \leq t^{-1 / p}$.

Proof. By Hölder's inequality,

$$
\left|\sum_{j=1+q_{n-1}}^{q_{n}} a_{j} w_{j}\right|^{p} \leq\left(\sum_{j=1+q_{n-1}}^{q_{n}} w_{j}\right)^{p-1} \sum_{j=1+q_{n-1}}^{q_{n}}\left|a_{j}\right|^{p} w_{j}, \quad n \in \mathbb{N} .
$$

Thus, if $f=\left(a_{j}\right)_{j=1}^{\infty}$,

$$
\begin{aligned}
\left\|P_{\gamma}(f)\right\|_{p}^{p} & \leq \sum_{n=1}^{\infty} \frac{\sum_{j=1}^{q_{n}-q_{n-1}} w_{j}}{\sum_{j=1+q_{n-1}}^{q_{n}} w_{j}} \sum_{j=1+q_{n-1}}^{q_{n}}\left|a_{j}\right|^{p} w_{j} \\
& \leq \frac{1}{t} \sum_{n=1}^{\infty} \sum_{j=1+q_{n-1}}^{q_{n}}\left|a_{j}\right|^{p} w_{j} \\
& =\frac{1}{t} \sum_{j=1}^{\infty}\left|a_{j}\right|^{p} w_{j} \\
& \leq \frac{1}{t}\|f\|_{g}^{p} .
\end{aligned}
$$


In our route to prove Theorem 2.1 we need to revisit a result from [6].

Proposition 2.7 (cf. [6, Proposition 3.2]). Let $1 \leq p<\infty$, w $\in \mathcal{W}$ and $t \in(0, \infty)$. Let $\mathcal{B}=\left(\mathbf{y}_{n}\right)_{n=1}^{\infty}$ be a block basic sequence of the unit vector basis of $g(\mathbf{w}, p)$ such that $\left\|\mathbf{y}_{n}\right\| \leq t$ for every $n \in \mathbb{N}$. Then $\mathcal{B}$ is $t$-dominated by the unit vector basis of $\ell_{p}$.

Proof. Although [6, Proposition 3.2] tackles only the case when $\left\|y_{n}\right\|_{g}=$ 1 , its proof can be reproduced almost verbatim in this slightly more general setting.

We are now in a position to complete the proof of Theorem 2.1.

Proof of Theorem 2.1. Let $\mathcal{D}=\left\{(i, n) \in \mathbb{N}^{2}: 1 \leq i \leq n\right\}$ and $t=$ $\sqrt{1+\varepsilon}$. Use Lemmas 2.5 and 2.2 to recursively construct $\kappa_{n}=\left(k_{i, n}\right)_{i=1}^{n}$, $n \in \mathbb{N}$, verifying $\left\|\mathbf{v}\left[\kappa_{n}\right]\right\|_{g} \leq t$ for all $n \in \mathbb{N}$ and

$$
\frac{\sum_{j=1+m_{n}}^{m_{n}+k_{i, n}} w_{j}}{\sum_{j=1}^{k_{i, n}} w_{j}} \geq t^{-p}
$$

for all $(i, n) \in \mathcal{D}$, where $m_{n}=\sum_{r=1}^{n-1} \max _{1 \leq i \leq k} k_{i, r}$ for $n \in \mathbb{N} \cup\{0\}$.

Denote, for $n \in \mathbb{N}$ and $0 \leq i \leq n$

$$
m_{i, n}=\sum_{r=1}^{n-1} \sum_{d=1}^{r} k_{d, r}+\sum_{d=1}^{i} k_{d, n}
$$

and put, for $(i, n) \in \mathcal{D}$,

$$
\begin{aligned}
& \text { - } J_{i, n}=\left\{n \in \mathbb{N}: 1+m_{i-1, n} \leq j \leq m_{i, n}\right\}, \\
& \text { - } \mathbf{y}_{i, n}=\left(\sum_{j=1}^{k_{i, n}} w_{j}\right)^{-1 / p} \sum_{j \in J_{i, n}} \mathbf{e}_{j}, \\
& \text { - } \mathbf{y}_{i, n}^{*}\left(\left(a_{j}\right)_{j=1}^{\infty}\right)=\left(\sum_{j=1}^{k_{i, n}} w_{j}\right)^{1 / p}\left(\sum_{j \in J_{i, n}} w_{j}\right)^{-1} \sum_{j \in J_{i, n}} w_{j} a_{j} .
\end{aligned}
$$

Note that $\left(J_{i, n}\right)_{(i, n) \in \mathcal{D}}$ is a partition $\mathbb{N}$, and it is straightforward to check that $\left(\mathbf{y}_{i, n}, \mathbf{y}_{i, n}^{*}\right)_{(i, n) \in \mathcal{D}}$ is a biorthogonal system. Thus, if we define $P: \mathbb{F}^{\mathbb{N}} \rightarrow \prod_{n=1}^{\infty} \mathbb{F}^{n}$ by

$$
P(f)=\left(\left(\mathbf{y}_{i, n}^{*}(f)\right)_{i=1}^{n}\right)_{n=1}^{\infty}, \quad f \in \mathbb{F}^{\mathbb{N}}
$$

and $S: \prod_{n=1}^{\infty} \mathbb{F}^{n} \rightarrow \mathbb{F}^{\mathbb{N}}$ by

$$
f=\left(\left(a_{i, n}\right)_{i=1}^{n}\right)_{n=1}^{\infty} \in \prod_{n=1}^{\infty} \mathbb{F}^{n} \mapsto S(f)=\sum_{n=1}^{\infty} \sum_{i=1}^{n} a_{i, n} \mathbf{y}_{i, n}
$$


we have $P \circ S=\operatorname{Id}_{\prod_{n=1}^{\infty} \mathbb{F}^{n}}$. Thus, the proof will be over once we show that

$$
\max \left\{\left\|P: g(\mathbf{w}, p) \rightarrow\left(\oplus_{n=1}^{\infty} \ell_{\infty}^{n}\right)_{p}\right\|,\left\|S:\left(\oplus_{n=1}^{\infty} \ell_{\infty}^{n}\right)_{p} \rightarrow g(\mathbf{w}, p)\right\|\right\} \leq t .
$$

Given $\alpha=\left(i_{n}\right)_{n=1}^{\infty}$ with $1 \leq i_{n} \leq n$, let $\phi_{\alpha} \in \mathcal{O}$ be defined by $\phi_{\alpha}(\mathbb{N})=K_{\alpha}:=\cup_{n=1}^{\infty} J_{i_{n}, n}$. Consider the operator

$$
V_{\alpha}: \mathbb{F}^{\mathbb{N}} \rightarrow \mathbb{F}^{\mathbb{N}}, \quad f=\left(a_{j}\right)_{j=1}^{\infty} \mapsto V_{\alpha}(f)=\left(a_{\phi_{\alpha}(j)}\right)_{j=1}^{\infty} .
$$

That is, $V_{\alpha}(f)$ is the sequence obtained by removing from $f$ its coefficients outside $K_{\alpha}$. The 1-subsymmetry of the unit vector basis of $g(\mathbf{w}, p)$ yields $\left\|V_{\alpha}: g(\mathbf{w}, p) \rightarrow g(\mathbf{w}, p)\right\| \leq 1$. Put $q_{n}(\alpha)=q_{n}:=$ $\sum_{r=1}^{n} k_{i_{r}, r}$ for $n \in \mathbb{N} \cup\{0\}$. Since $\mathbf{w}$ is non-increasing,

$$
\frac{\sum_{j=1+q_{n-1}}^{q_{n}} w_{j}}{\sum_{j=1}^{k_{i_{n}, n}} w_{j}} \geq \frac{\sum_{j=1+m_{n}}^{m_{n}+k_{i_{n}, n}} w_{j}}{\sum_{j=1}^{k_{i_{n}, n}} w_{j}} \geq t^{-p}, \quad n \in \mathbb{N} .
$$

Therefore, by Lemma 2.6,

$$
\left(\sum_{n=1}^{\infty}\left|\mathbf{y}_{i_{n}, n}^{*}(f)\right|^{p}\right)^{1 / p} \leq t\left\|V_{\alpha}(f)\right\|_{g} \leq t\|f\|_{g}, \quad f \in \mathbb{F}^{\mathbb{N}} .
$$

Taking the supremum on all possible choices of $\left(i_{n}\right)_{n=1}^{\infty}$ we obtain

$$
\|P(f)\|_{\left(\oplus_{n=1}^{\infty} \ell_{\infty}^{n}\right)_{p}}=\left(\sum_{n=1}^{\infty}\left(\max _{1 \leq i \leq n}\left|\mathbf{y}_{i, n}^{*}(f)\right|\right)^{p}\right)^{1 / p} \leq t\|f\|_{g}, \quad f \in \mathbb{F}^{\mathbb{N}} .
$$

Let $\mathbf{y}_{n}=\sum_{i=1}^{n} \mathbf{y}_{i, n}$ for $n \in \mathbb{N}$. We have that $\left(\mathbf{y}_{n}\right)_{n=1}^{\infty}$ is a block basic sequence of the unit vector system and that $\mathbf{y}_{n}=\mathrm{I}_{m_{i-1, n}}\left(\mathbf{v}\left[\kappa_{n}\right]\right)$. Consequently, $\left\|\mathbf{y}_{n}\right\|_{g} \leq t$ for all $n \in \mathbb{N}$. If $f=\left(\left(a_{i, n}\right)_{i=1}^{n}\right)_{n=1}^{\infty} \in \prod_{n=1}^{\infty} \mathbb{F}^{n}$, invoking Proposition 2.7 and the 1-unconditionality of the unit vector system we obtain

$$
\begin{aligned}
\|S(f)\|_{g} & \leq\left\|\sum_{n=1}^{\infty}\left(\sup _{1 \leq i \leq n}\left|a_{i, n}\right|\right)\left(\sum_{i=1}^{n} \mathbf{y}_{i, n}\right)\right\|_{g} \\
& =\left\|\sum_{n=1}^{\infty}\left(\max _{1 \leq i \leq n}\left|a_{i, n}\right|\right) \mathbf{y}_{n}\right\|_{g} \\
& \leq t\left(\sum_{n=1}^{\infty}\left(\max _{1 \leq i \leq n}\left|a_{i, n}\right|\right)^{p}\right)^{1 / p} \\
& =t\|f\|_{\left(\oplus_{n=1}^{\infty} \ell_{\infty}^{n}\right)_{p}},
\end{aligned}
$$

as desired. 


\section{Conditional bases in Garling Sequence spaces}

In 1964, Pełczyński and Singer proved that every Banach space with a basis has a conditional (i.e., not unconditional) basis [19]. Thus in order to get a more accurate information on a given space by means of conditional bases, one needs to restrict the discussion on their existence by imposing certain distinctive properties.

One way to specify a special property on conditional bases is precisely by quantifying their conditionality. In order to do that we consider the sequences $\left(k_{m}[\mathcal{B}, \mathbb{X}]\right)_{m=1}^{\infty}$ and $\left(L_{m}[\mathcal{B}, \mathbb{X}]\right)_{m=1}^{\infty}$ defined by

$$
\begin{aligned}
& k_{m}[\mathcal{B}]=k_{m}[\mathcal{B}, \mathbb{X}]=\sup \left\{\frac{\left\|S_{A}(f)\right\|}{\|f\|}:|A| \leq m, A \subseteq \mathbb{N}\right\}, \\
& L_{m}[\mathcal{B}]=L_{m}[\mathcal{B}, \mathbb{X}]=\sup \left\{\frac{\left\|S_{A}(f)\right\|}{\|f\|}: \operatorname{supp}(f) \subseteq[1, m], A \subseteq \mathbb{N}\right\} .
\end{aligned}
$$

Indeed, since a basis $\mathcal{B}$ is unconditional if and only if $\sup _{m} L_{m}[\mathcal{B}]<\infty$ or $\sup _{m} k_{m}[\mathcal{B}]<\infty$, the growth of any of those sequences can be interpreted as a measure of the conditionality of $\mathcal{B}$. The gauge $k_{m}[\mathcal{B}]$ does not depend on the way in which the vectors of the basis are arranged, and so is arguably more natural than $L_{m}[\mathcal{B}]$. However, the sequence $L_{m}[\mathcal{B}]$ introduced in [8] has shown to be in some settings a more accurate tool for studying conditional bases (see also [3]). Note

Plantear la pregunta en los términos exactos en los que se responde a continuación es poco elegante that $L_{m}[\mathcal{B}] \leq k_{m}[\mathcal{B}]$

For every basis $\mathcal{B}$ in a Banach space $\mathbb{X}$ one always has the estimate $k_{m}[\mathcal{B}, \mathbb{X}] \lesssim m$, for $m \in \mathbb{N}$. Conversely, it is known (see [8, Theorem $3.5]$ ) that $\mathbb{X}$ is not superreflexive if and only if there is a basic sequence $\mathcal{B}^{\prime}$ in $\mathbb{X}$ with $m \lesssim L_{m}\left[\mathcal{B}^{\prime}, \mathbb{X}\right]$ for every $m$ for $m \in \mathbb{N}$. Hence it is natural to wonder if, being non-superreflexive, $g(\mathbf{w}, p)$ will possess a conditional basis $\mathcal{B}$ with $L_{m}[\mathcal{B}, g(\mathbf{w}, p)] \approx m$ every $m$. Hence it is natural to wonder if, being non-superreflexive, $g(\mathbf{w}, p)$ will possess not only a basic sequence but a basis of the whole space with this property. The answer is positive as we next show.

Proposition 3.1. Let $1 \leq p<\infty$ and $\mathbf{w} \in \mathcal{W}$. Then $g(\mathbf{w}, p)$ has a (conditional) basis $\mathcal{B}$ with $L_{m}[\mathcal{B}] \approx m$ for $m \in \mathbb{N}$.

Proof. It is known that the summing system $\left(\mathbf{s}_{j}\right)_{j=1}^{\infty}$ given by

$$
\mathbf{s}_{j}=\sum_{k=1}^{j} \mathbf{e}_{j}
$$

is a basis for $c_{0}$ such that $\operatorname{span}\left(\mathbf{s}_{j}: 1 \leq j \leq n\right)=\ell_{\infty}^{n}$ for all $n \in$ $\mathbb{N}$ with $L_{m}[\mathcal{S}] \approx m$ for $m \in \mathbb{N}$ (see, e.g., [3, Lemma 4.9]). Hence, $\mathcal{B}_{0}:=\bigoplus_{n=1}^{\infty}\left(\mathbf{s}_{j}\right)_{j=1}^{2^{n}}$ is a basis for $\mathbb{X}:=\left(\oplus_{n=1}^{\infty} \ell_{\infty}^{2^{n}}\right)_{p}$ with $L_{m}\left[\mathcal{B}_{0}\right] \approx m$ 
for $m \in \mathbb{N}$ (see [3, Lemma 2.3]). Then $\mathcal{B}:=\mathcal{B}_{0} \oplus\left(\mathbf{e}_{j}\right)_{j=1}^{\infty}$ is a basis for $\mathbb{Y}=\mathbb{X} \oplus g(\mathbf{w}, p)$ with $L_{m}[\mathcal{B}] \approx m$ for $m \in \mathbb{N}$ (see [3, Lemma 2.2]). Finally, since $\mathbb{X} \oplus\left(\oplus_{n=1}^{\infty} \ell_{\infty}^{n}\right)_{p} \approx\left(\oplus_{n=1}^{\infty} \ell_{\infty}^{n}\right)_{p}$ (see, e.g., [2, Appendix 4.1]), Theorem 2.1 yields $\mathbb{Y} \approx g(\mathbf{w}, p)$.

Now we will look into conditional bases in Garling sequence spaces that have some special features in relation to the optimality of the greedy algorithm. For the convenience of the reader we recall the relevant concepts from approximation theory, thus making our exposition self-contained.

Let $\mathcal{B}=\left(\mathbf{x}_{n}\right)_{n=1}^{\infty}$ be a basis for a Banach space $\mathbb{X}$. A finite set $G \subseteq \mathbb{N}$ is said to be a greedy set for $f=\sum_{n=1}^{\infty} a_{n} \mathbf{x}_{n} \in \mathbb{X}$ if $\left|a_{n}\right| \geq\left|a_{j}\right|$ whenever $n \in G$ and $j \in \mathbb{N} \backslash G$. A greedy sum will be a coordinate projection on a greedy set. The basis $\mathcal{B}$ is said to be $C$-greedy if greedy sums provide, up to a multiplicative constant $C \in[1, \infty)$, optimal approximations amongst linear combinations with respect to the basis, i.e.,

$$
\left\|f-S_{G}(f)\right\| \leq C\left\|f-\sum_{j \in A} c_{j} \mathbf{x}_{j}\right\|
$$

whenever $G$ is a greedy set for $f \in \mathbb{X},|G|=|A|$, and $\left(c_{j}\right)_{j \in A} \in \mathbb{F}^{A}$.

Being less demanding, a basis $\mathcal{B}$ is said to be almost greedy if the greedy sums (essentially) provide the optimal approximations amongst coordinate projections, that is, there is a constant $C<\infty$ such that whenever $G$ is a greedy set for $f \in \mathbb{X}$ and $|G|=|A|$,

$$
\left\|f-S_{G}(f)\right\| \leq C\left\|f-S_{A}(f)\right\| .
$$

Almost greedy bases enjoy the property of being democratic (see [14, Theorem 3.3]), i.e., there is a sequence $\left(\lambda_{m}\right)_{m=1}^{\infty}$ such that for any finite subset $A$ of $\mathbb{N}$,

$$
\left\|\sum_{j \in A} \varepsilon_{j} \mathbf{x}_{j}\right\| \approx \lambda_{|A|} .
$$

In this case, $\left(\lambda_{m}\right)_{m=1}^{\infty}$ is equivalent to the fundamental function $\varphi_{m}[\mathcal{B}, \mathbb{X}]$ of $\mathcal{B}$ defined by

$$
\varphi_{m}[\mathcal{B}, \mathbb{X}]=\sup _{|A| \leq m}\left\|\sum_{j \in A} \mathbf{x}_{j}\right\|, \quad m \in \mathbb{N} .
$$

Note that the unit vector basis $\mathcal{E}=\left(\mathbf{e}_{j}\right)_{j=1}^{\infty}$ of $g(\mathbf{w}, p)$ verifies

$$
\left\|\sum_{j \in A} \mathbf{e}_{j}\right\|_{g}=\left(\sum_{j=1}^{|A|} w_{j}\right)^{1 / p}
$$

Ahora sobra la definición de base greedy 
for all $A \subseteq \mathbb{N}$ finite and so

$$
\varphi_{m}[\mathcal{E}, g(\mathbf{w}, p)]=\left(\sum_{j=1}^{m} w_{j}\right)^{1 / p}, \quad m \in \mathbb{N} .
$$

When a basis $\mathcal{B}$ of a Banach space $\mathbb{X}$ is almost greedy, the size of the members of the sequence $\left(k_{m}[\mathcal{B}, \mathbb{X}]\right)_{m=1}^{\infty}$ is controlled by a slowly growing function to the extent that (see [14, Lemma 8.2])

$$
k_{m}[\mathcal{B}] \lesssim \log m, \quad m \geq 2 .
$$

Moreover, by [4, Theorem 1.1] this inequality its optimal only if $\mathbb{X}$ is not superreflexive.

Our last result in this note is a new addition to the subject of finding (non-superreflexive) spaces possessing almost greedy conditional bases for which the estimate (3.1) is optimal, i.e., $k_{m}[\mathcal{B}] \approx \log m$ for $m \geq 2$. This topic was initiated by Garrigós et al. in [17] and has been given continuity through several papers and authors (see $[3,4,8,11,18]$ ).

The proof of Theorem 3.2 leans of two regularity properties enjoyed by some weights whose definitions we refresh. Following [15], a weight $\left(\lambda_{m}\right)_{m=1}^{\infty}$ is said to have the lower regularity property (LRP for short) if there is a positive integer $b$ such that

$$
2 \lambda_{m} \leq \lambda_{b m} . \quad m \in \mathbb{N}
$$

A weight $\left(\lambda_{m}\right)_{m=1}^{\infty}$ is said to have the upper regularity property (URP for short) if there is an integer $b \geq 3$ such that

$$
\lambda_{b m} \leq \frac{b}{2} \lambda_{m}, \quad m \in \mathbb{N} .
$$

Theorem 3.2. Let $1 \leq p<\infty$ and $\mathbf{w} \in \mathcal{W}$.

(a) There is an almost greedy basis $\mathcal{B}$ for $g(\mathbf{w}, p)$ with fundamental function equivalent to $\left(m^{1 / p}\right)_{m=1}^{\infty}$ such that $L_{m}[\mathcal{B}] \approx \log m$ for $m \geq 2$.

(b) If the weight $\mathbf{w}$ is bi-regular there is an almost greedy basis $\mathcal{B}$ for $g(\mathbf{w}, 1)$ with fundamental function equivalent to $\left(\sum_{j=1}^{m} w_{j}\right)_{m=1}^{\infty}$ such that $L_{m}[\mathcal{B}] \approx \log m$ for $m \geq 2$.

(c) If $\mathbf{w}$ is regular and $p>1$, there is an almost greedy basis $\mathcal{B}$ for $g(\mathbf{w}, p)$ with fundamental function equivalent to the sequence $\left.\left(\sum_{j=1}^{m} w_{j}\right)^{1 / p}\right)_{m=1}^{\infty}$ such that $L_{m}[\mathcal{B}] \approx \log m$ for $m \geq 2$.

Proof. Applying [3, Theorem 4.1] with $\mathbb{S}=\ell_{p}$, and taking into account Theorem 3.1, gives a basis $\mathcal{B}$ as claimed in (a) for the the Banach space $\ell_{p} \oplus g(\mathbf{w}, p) \approx g(\mathbf{w}, p)$. 
Assume that $\mathbf{w}$ is regular. Then, by [7, Proposition 2.5], its primitive weight $\left(\sum_{j=1}^{m} w_{j}\right)_{m=1}^{\infty}$ has the LRP. Therefore $\left(\left(\sum_{j=1}^{m} w_{j}\right)^{1 / p}\right)_{m=1}^{\infty}$ also has the LRP for any $1 \leq p<\infty$. Note also that $m w_{m} \approx \sum_{j=1}^{n} w_{j}$ and so the conjugate weight $\mathbf{w}^{*}$ is equivalent to $1 /\left(\sum_{j=1}^{m} w_{j}\right)_{m=1}^{\infty}$. Consequently, [1, Lemma 2.12 (iii)] yields that $\left(\sum_{j=1}^{m} w_{j}\right)_{m=1}^{\infty}$ has the URP whenever $\mathbf{w}^{*}$ is bi-regular. Since $m^{-1}\left(\sum_{j=1}^{m} w_{j}\right)_{m=1}^{\infty}$ is non-increasing, in the case when $p>1$, [1, Lemma 2.12 (ii)] yields $\left(\left(\sum_{j=1}^{m} w_{j}\right)^{1 / p}\right)_{m=1}^{\infty}$ has the URP.

Note that [3, Theorem 4.1] holds replacing the hypothesis " $\mathbb{S}$ has nontrivial type" with the hypothesis "the fundamental function of the subsymmetric basis of $\mathbb{S}$ has both the LRP and the URP". Hence, under the assumptions in both (b) and (c), applying [3, Theorem 4.1] with $\mathbb{S}=g(\mathbf{w}, p)$ gives a basis as desired for the Banach space $g(\mathbf{w}, p) \oplus$ $g(\mathbf{w}, p) \approx g(\mathbf{w}, p)$.

\section{REFERENCES}

[1] F. Albiac and J. L. Ansorena, Lorentz spaces and embeddings induced by almost greedy bases in Banach spaces, Constr. Approx. 43 (2016), no. 2, 197-215.

[2] I Isomorphic classification of mixed sequence spaces and of Besov spaces over $[0,1]^{d}$, Math. Nachr. 290 (2017), no. 8-9, 1177-1186.

[3] F. Albiac, J. L. Ansorena, S. J. Dilworth, and Denka Kutzarova, Building highly conditional almost greedy and quasi-greedy bases in Banach spaces, arXiv:1803.08351.

[4] F. Albiac, J. L. Ansorena, G. Garrigós, E. Hernández, and M. Raja, Conditionality constants of quasi-greedy bases in super-reflexive Banach spaces, Studia Math. 227 (2015), no. 2, 133-140.

[5] F. Albiac, J. L. Ansorena, D. Leung, and B. Wallis, Optimality of the rearrangement inequality with applications to Lorentz-type sequence spaces, Math. Inequal. Appl. 21 (2018), no. 1, 127-132.

[6] F. Albiac, J.L. Ansorena, and B. Wallis, Garling sequence spaces, J. London Math. Soc., DOI 10.1112/jlms.12129.

[7] F. Albiac, J. L. Ansorena, and B. Wallis, 1-greedy renormings of Garling sequence spaces, J. Approx. Theory 230 (2018), 13-23.

[8] F. Albiac, J. L. Ansorena, and P. Wojtaszczyk, Conditionality constants of quasi-greedy bases in non-superreflexive Banach space, Constr. Approx., DOI 10.1007/s00365-017-9399-x.

[9] F. Albiac and N. J. Kalton, Topics in Banach space theory, 2nd revised and updated edition, Graduate Texts in Mathematics, vol. 233, Springer International Publishing, 2016.

[10] Z. Altshuler, Uniform convexity in Lorentz sequence spaces, Israel J. Math. 20 (1975), no. 3-4, 260-274.

[11] P. Berná, O. Blasco, G. Garrigós, E. Hernández, and T. Oikhberg, The greedy algorithm for non-greedy type bases, Submitted.

[12] Z. Altshuler, P. G. Casazza, and Bor Luh Lin, On symmetric basic sequences in Lorentz sequence spaces, Israel J. Math. 15 (1973), 140-155. 
14 F. ALBIAC, J. L. ANSORENA, S. J. DILWORTH, AND DENKA KUTZAROVA

[13] P. G. Casazza and B.L. Lin, On symmetric basic sequences in Lorentz sequence spaces. II, Israel J. Math. 17 (1974), 191-218.

[14] S. J. Dilworth, N. J. Kalton, and Denka Kutzarova, On the existence of almost greedy bases in Banach spaces, Studia Math. 159 (2003), no. 1, 67-101.

[15] S. J. Dilworth, N. J. Kalton, Denka Kutzarova, and V. N. Temlyakov, The thresholding greedy algorithm, greedy bases, and duality, Constr. Approx. 19 (2003), no. 4, 575-597.

[16] D. J. H. Garling, Symmetric bases of locally convex spaces, Studia Math. 30 (1968), 163-181.

[17] G. Garrigós, E. Hernández, and T. Oikhberg, Lebesgue-type inequalities for quasi-greedy bases, Constr. Approx. 38 (2013), no. 3, 447-470.

[18] G. Garrigós and P. Wojtaszczyk, Conditional quasi-greedy bases in Hilbert and Banach spaces, Indiana Univ. Math. J. 63 (2014), no. 4, 1017-1036.

[19] A. Pełczyński and I. Singer, On non-equivalent bases and conditional bases in Banach spaces, Studia Math. 25 (1964/1965), 5-25.

Mathematics Department-Inamat, Universidad Pública de Navarra, PAMPLONA 31006, SPAIN

E-mail address: fernando.albiac@unavarra.es

Department of Mathematics and Computer Sciences, Universidad De LA Rioja, Logroño 26004, SpAin

E-mail address: joseluis.ansorena@unirioja.es

Department of Mathematics, University of South Carolina, Columbia SC 29208, USA

E-mail address: dilworth@math.sc.edu

Department of MAThematics University of ILlinois at URBAnA-Champaign, Urbana, IL 61801, USA, And Institute of MAthematics, Bulgarian ACADEMY OF SCIEnCES, Sofia, Bulgaria.

E-mail address: denka@math.uiuc.edu 